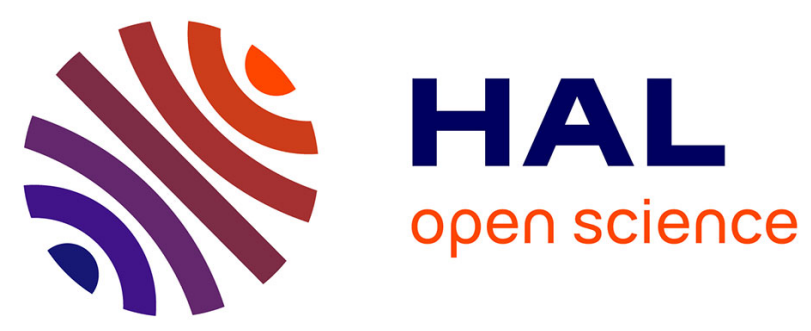

\title{
3D Reconstruction from SECCHI-EUVI Images Using an Optical-Flow Algorithm: Method Description and Observation of an Erupting Filament
}

Samuel Gissot, Jean-François Hochedez, Pierre Chainais, Jean-Pierre Antoine

\section{- To cite this version:}

Samuel Gissot, Jean-François Hochedez, Pierre Chainais, Jean-Pierre Antoine. 3D Reconstruction from SECCHI-EUVI Images Using an Optical-Flow Algorithm: Method Description and Observation of an Erupting Filament. Solar Physics, 2008, 252 (2), pp.397-408. hal-00808611

\section{HAL Id: hal-00808611 https://hal.science/hal-00808611}

Submitted on 9 Apr 2013

HAL is a multi-disciplinary open access archive for the deposit and dissemination of scientific research documents, whether they are published or not. The documents may come from teaching and research institutions in France or abroad, or from public or private research centers.
L'archive ouverte pluridisciplinaire HAL, est destinée au dépôt et à la diffusion de documents scientifiques de niveau recherche, publiés ou non, émanant des établissements d'enseignement et de recherche français ou étrangers, des laboratoires publics ou privés. 


\title{
3D Reconstruction from SECCHI-EUVI Images Using an Optical-Flow Algorithm: Method Description and Observation of an Erupting Filament
}

\author{
S.F. Gissot ${ }^{1,2}$, J.-F. Hochedez ${ }^{1}$, P. Chainais $^{3}$, \\ J.-P. Antoine ${ }^{2}$ \\ Received ; accepted \\ (C) Springer
}

\begin{abstract}
SECCHI-EUVI telescopes provide the first EUV images enabling a $3 \mathrm{D}$ reconstruction of solar coronal structures. We present a stereoscopic reconstruction method based on the Velociraptor algorithm, a multiscale optical-flow method that estimates displacement maps in sequences of EUV images. Following earlier calibration on sequences of SoHO-EIT data, we apply the algorithm to retrieve depth information from the two STEREO viewpoints using the SECCHIEUVI telescope. We first establish a simple reconstruction formula that gives the radial distance to the centre of the Sun of a point identified both in EUVI-A and EUVI-B from the separation angle and the displacement map. We select pairs of images taken in the $30.4 \mathrm{~nm}$ passband of EUVI-A and EUVI-B, and apply a rigid transform from the EUVI-B image in order to set both images in the same frame of reference. The optical flow computation provides displacement maps from which we reconstruct a dense map of depths using the stereoscopic reconstruction formula. Finally we discuss the estimation of the height of an erupting filament.
\end{abstract}

Keywords: Chromosphere: Active, Prominences: Dynamics, Formation and Evolution, Technique: 3D reconstruction, Stereoscopy, Optical Flow

\section{Introduction}

The complexity of magnetic structures of the solar corona is well observed in EUV observations of the Sun. In EUV images of the solar atmosphere, loops and filaments appear as compact structures that are well observed at the limb and

\footnotetext{
1 Solar Influences Data analysis Centre, Royal Observatory of Belgium, Avenue Circulaire 3, B-1180 Brussels, Belgium (e-mail: samuel.gissot@sidc.be, hochedez@sidc.be)

${ }^{2}$ Unité de Physique Théorique et de Physique Mathématique - FYMA, Université catholique de Louvain, Chemin du Cyclotron 2, B-1348 Louvain-la-Neuve, Belgium 3 LIMOS, Université Blaise Pascal Clermont II, 63173 Aubière CEDEX, France
} 
on the disc when compared to the surrounding fuzzy corona. In the latter case, there remains an ambiguity on the height of the features. In order to solve the third dimension, two points of view are necessary to perform a stereoscopic reconstruction. The height of EUV bright points has been estimated and analyzed in Brajša et al. (2004) using the method proposed by Rosa et al. (1998). Similar work has been achieved on filament height estimation (Vršnak et al., 1999). Since the launch of the STEREO mission (Kaiser et al., 2007), it is possible to resolve the $3 \mathrm{D}$ ambiguity by using the observation of the two viewpoints STEREO-A and STEREO-B. First loop reconstructions have been published in Feng et al. (2007) using a loop extraction (Inhester et al., 2007). A method for stereoscopic reconstruction and epipolar geometry has been proposed (Inhester, 2006). We present a method for retrieving the depth information from a pair of STEREO-SECCHI-EUVI images observed on-board the two spacecrafts of the STEREO mission using simple geometrical facts and a novel algorithm, Velociraptor (Gissot et al., 2003; Gissot and Hochedez, 2007), to estimate the subpixel displacement. The Velociraptor algorithm is a multiscale optical flow algorithm derived from a local gradient-based technique able to estimate displacement and brightness variation maps in solar extreme-ultraviolet images as recorded by the Extreme ultraviolet Imaging Telescope (EIT) on board the Solar and Heliospheric Observatory (SoHO) and by the Transition Region and Coronal Explorer (TRACE). Here the displacement maps to estimate are shifts along the epipolar line caused by the separation of the two STEREO viewpoints, and no brightness variation is supposed: this method is here applied to estimate the height of an erupting filament.

\section{3D Reconstruction Method}

In this section we present the method for retrieving the altitude information from a pair of STEREO images. In the following we will refer to the image observed in EUVI on-board STEREO spacecraft STEREO-A and STEREO-B as EUVI-A and EUVI-B, respectively. We introduce two heliocentric-Cartesian coordinate systems $S_{\mathrm{A}}$ and $S_{\mathrm{B}}$ with notation $(x, y, z)$, that both have their origin at the Sun centre $O$, with $y$ normal to the STEREO mission plane (STEREOA,STEREO-B,O) and $z$ pointing to solar disc centre of either EUVI-A (system $S_{\mathrm{A}}$ ), or EUVI-B (system $S_{\mathrm{B}}$ ). In the following, the index A (resp. B) refers to the coordinate system $S_{\mathrm{A}}$ (resp. $S_{\mathrm{B}}$ ). The objective is to measure the radius $R=O P$ of a point $P$ of coordinates $\left(X_{\mathrm{A}}, Y_{\mathrm{A}}, Z_{\mathrm{A}}\right)$ in $S_{\mathrm{A}}$ and $\left(X_{\mathrm{B}}, Y_{\mathrm{B}}, Z_{\mathrm{B}}\right)$ in $S_{\mathrm{B}}$. After the pre-registration step, the point $P$ observed in EUVI-A is seen in EUVI-B as $P^{\prime}$, i.e. as $P$ rotated by an angle of $\Delta \lambda$.

\subsection{Pre-registration}

Since the Sun may be assumed to be at an infinite distance of STEREO-A and of STEREO-B, Figure 3 shows that in both instruments the solar disc as traced by its limb is the orthogonal projection of the solar sphere of centre $O$ and photospheric radius $R_{\mathrm{s}}$ as observed in EUVI-A. In the following, the separation 


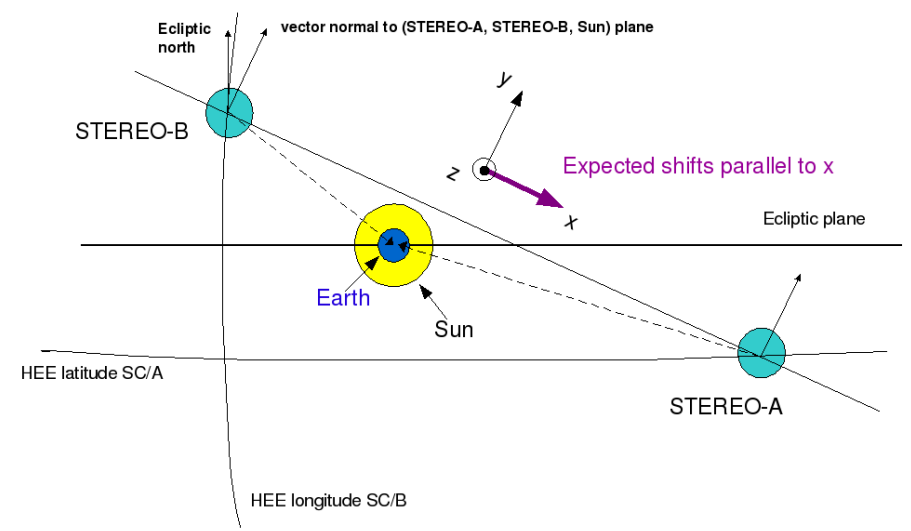

Figure 1. STEREO mission plane (STEREO-A, STEREO-B, O) and ecliptic north axis. The $S_{\mathrm{A}}$ and $S_{\mathrm{B}}$ coordinate systems have their origin at the Sun centre $O$ and their $y$-axis perpendicular to (STEREO-A, STEREO-B, $O$ ) and $z$ pointing either to STEREO-A $\left(S_{\mathrm{A}}\right)$ or STEREO-B $\left(S_{\mathrm{B}}\right)$.

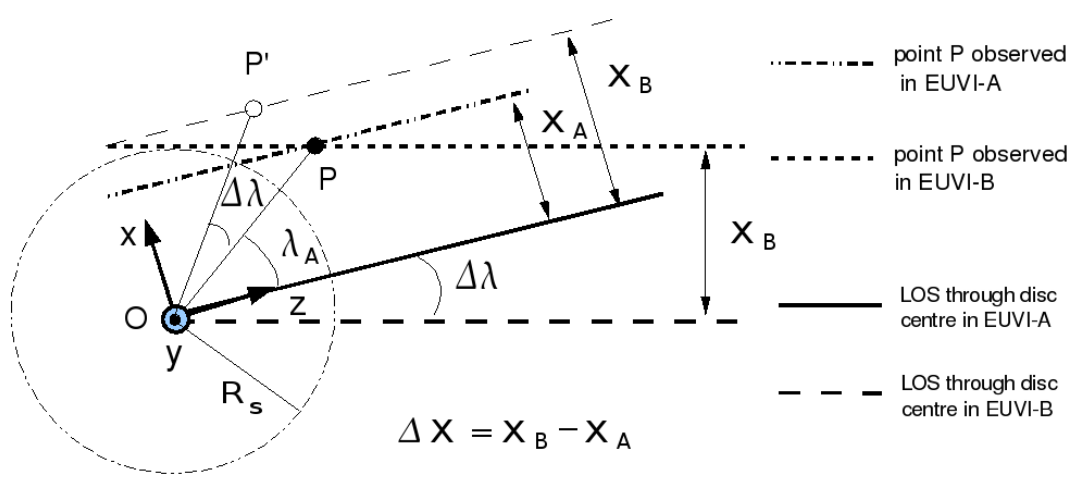

Figure 2. Apparent displacement $\Delta X$ of $P$ after the pre-registration step applied to EUVI-B so that it can be matched with EUVI-A. The point $P$ appears as $P^{\prime}$ in EUVI-B where $P^{\prime}$ is the point $P$ rotated by $\Delta \lambda$.

angle between spacecrafts is denoted by $\Delta \lambda, \lambda_{\mathrm{A}}$ is the angle of $P$ to $O y, R$ is the distance of $P$ to the Sun centre, $\left(X_{\mathrm{A}}, Y_{\mathrm{A}}, Z_{\mathrm{A}}\right)$ (resp. $\left.\left(X_{\mathrm{B}}, Y_{\mathrm{B}}, Z_{\mathrm{B}}\right)\right)$ are the coordinates of $P$ in $S_{\mathrm{A}}$ (resp. $S_{\mathrm{B}}$ ) (see Figure 4). Figure 2 shows that observing $P$ on the solar sphere from two different viewpoints (spacecrafts STEREO-A and STEREO-B) is equivalent to observing, from one unique viewpoint, the point $P$ in EUVI-A rotated to a virtual point $P^{\prime}$ by an angle $\Delta \lambda$. Indeed the displacement

$$
\Delta X=X_{\mathrm{B}}-X_{\mathrm{A}}=r \sin \left(\lambda_{\mathrm{A}}+\Delta \lambda\right)-r \sin \left(\lambda_{\mathrm{A}}\right),
$$




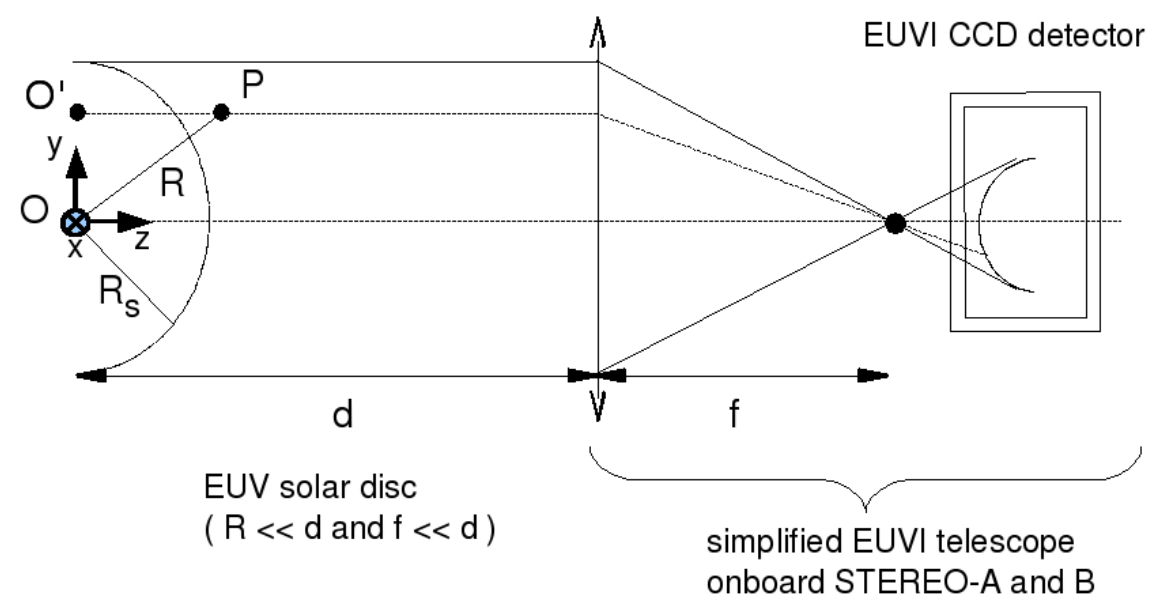

Figure 3. The projection model of the sun in the EUVI detectors of the STEREO spacecrafts STEREO-A and STEREO-B.

is equal to the apparent displacement of $P$ moving to $P^{\prime}$ after a rotation of angle $\Delta \lambda$ and centre $O$ (see Figure 4). Observed in EUVI-B, the point $P$ is equal to $P^{\prime}$ of coordinates $\left(X_{\mathrm{B}}, Y_{\mathrm{B}}, Z_{\mathrm{B}}\right)$ in $S_{\mathrm{A}}$.

This equivalence requires three conditions: the pointing to the heliospheric centre $O$ must be known with precision, the radius of the solar limb must be the same in EUVI-A and EUVI-B (or equivalently, the distance from STEREO-A and STEREO-B to $O$ must be known with precision), and the axis of the rotation, or the normal to the STEREO mission plane containing STEREO-A, STEREO$\mathrm{B}$ and $O$ (see Figure 1) must be known as well. If we impose that this axis be vertical in both image planes, the last requirement ensures that the epipolar lines are horizontal if we neglect the projective geometry effects, so that $Y_{\mathrm{A}}=Y_{\mathrm{B}}$. The pre-registration step consists in transforming EUVI-B using translation, rotation, and dilation, in order to set EUVI-B in the same reference frame as EUVI-A so that both image centres are pointing to the Sun centre, they both have the same apparent photospheric radius $R_{\mathrm{s}}$, and their vertical axis is aligned with the epipolar north. After applying the pre-registration procedure that reads the information contained in the FITS headers, EUVI-A and B now have:

- the line-of-sight (LOS) of the image centres passing through the Sun centre $O$ (after a translation),

- the vertical axis perpendicular to the plane containing (STEREO-A, STEREO-B, O), see Figure 1 (after a rotation),

- the same limb radius equal to the photospheric radius $R_{\mathrm{s}}$ from EUVI-A (after a dilation). This dilation is achieved using the distance to the Sun of both spacecrafts. 


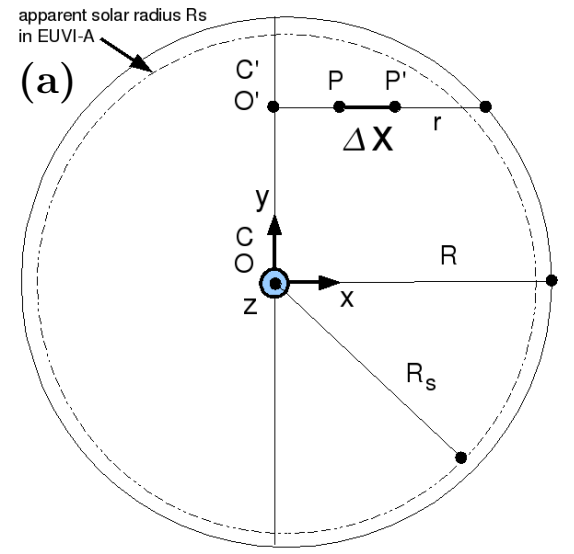

(b)

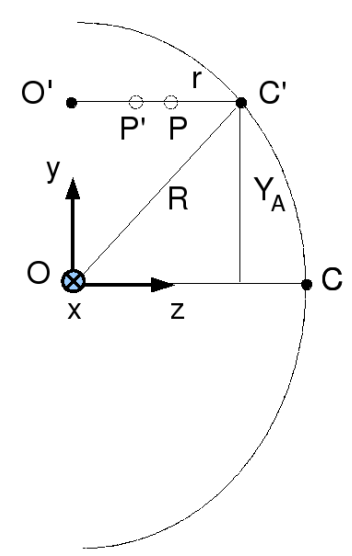

(c)

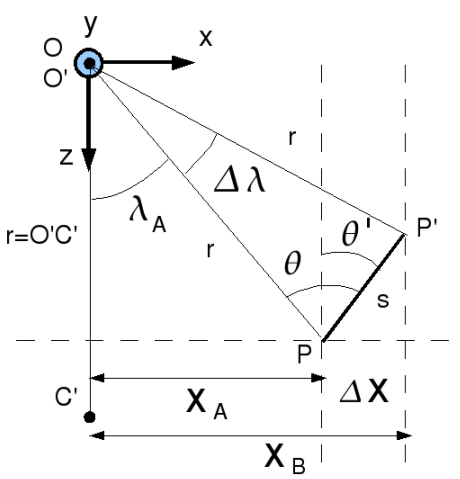

Figure 4. Representation of the angles $\lambda_{\mathrm{A}}, \Delta \lambda, \theta$, the radii $r$ and $R$, and the distance $s . R_{\mathrm{S}}$ is the photospheric radius as observed in EUVI-A. After the pre-registration step, both images EUVI-A and $-B$ are in the same frame of reference. Figure (a): front view from STEREO-A (EUVI-A) image. Figure (b): left view. Figure (c): top view.

\subsection{D Reconstruction Formula}

In Figure 4 the point $P$ becomes $P^{\prime}$ after a rotation of the sphere by an angle $\Delta \lambda$. The line $O C$ shows the LOS passing through the apparent disc centre (Figure 2 ) and pointing to the heliospheric centre $O$, which is now the same in EUVI-A and EUVI-B. As we estimate depths in EUVI-A, we denote by $\left(X_{\mathrm{A}}, Y_{\mathrm{A}}, Z_{\mathrm{A}}\right)$ the coordinates of $P$. Figure 4 introduces the angle $\lambda_{\mathrm{A}}$ and the distance $s$. In Figure 4 , we define $r^{2}=X_{\mathrm{A}}^{2}+Z_{\mathrm{A}}^{2}$ and $R^{2}=X_{\mathrm{A}}^{2}+Y_{\mathrm{A}}^{2}+Z_{\mathrm{A}}^{2}$.

As $O^{\prime} P=O^{\prime} P^{\prime}=r$, we have that

$$
s=2 r \sin (\Delta \lambda / 2)
$$

and

$$
\theta=\pi / 2-\Delta \lambda / 2
$$


Moreover, Figure 4 (bottom right) shows that $\theta^{\prime}=\theta-\lambda_{\mathrm{A}}$ and

$$
\sin \left(\theta^{\prime}\right)=\frac{\Delta X}{s}=\sin \left(\theta-\lambda_{\mathrm{A}}\right) .
$$

As a consequence, the expression of the displacement $\Delta X$ in terms of the angles $\lambda_{\mathrm{A}}$ and $\Delta \lambda$ is

$$
\Delta X=s \sin \left(\pi / 2-\lambda_{\mathrm{A}} / 2-\Delta \lambda / 2\right) .
$$

Using Equations (1) and (2), the displacement $\Delta X$ is related to $r$ and the angles $\lambda_{\mathrm{A}}$ and $\Delta \lambda$ by

$$
\Delta X=2 r \sin (\Delta \lambda / 2) \cos \left(\Delta \lambda / 2+\lambda_{\mathrm{A}}\right) .
$$

Furthermore, as $r^{2}=X_{\mathrm{A}}^{2}+r^{2} \cos \left(\lambda_{\mathrm{A}}\right)^{2}$ and

$$
X_{\mathrm{A}}=r \sin \left(\lambda_{\mathrm{A}}\right)=\sqrt{R^{2}-Y_{\mathrm{A}}^{2}} \sin \left(\lambda_{\mathrm{A}}\right),
$$

we have

$$
\Delta X=2 r \sin (\Delta \lambda / 2) \cos \left(\Delta \lambda / 2+\sin ^{-1}\left(X_{\mathrm{A}} / \sqrt{R^{2}-Y_{\mathrm{A}}^{2}}\right)\right)
$$

We can expand the right-hand side of Equation (3) since

$$
\begin{aligned}
r \cos \left(\Delta \lambda / 2+\lambda_{\mathrm{A}}\right) & =r \cos (\Delta \lambda / 2) \cos \left(\lambda_{\mathrm{A}}\right)-r \sin (\Delta \lambda / 2) \sin \left(\lambda_{\mathrm{A}}\right), \\
& =\cos (\Delta \lambda / 2)\left(r^{2}-X_{\mathrm{A}}^{2}\right)^{1 / 2}-\sin (\Delta \lambda / 2) X_{\mathrm{A}} .
\end{aligned}
$$

Injecting Equation (6) into Equation (3) gives

$$
r^{2}=X_{\mathrm{A}}^{2}+\left[\tan \left(\frac{\Delta \lambda}{2}\right) X_{\mathrm{A}}+\frac{\Delta X}{\sin \Delta \lambda}\right]^{2}
$$

for the value of the apparent radius $r$. As $r$ is equal to $O^{\prime} C^{\prime}=O^{\prime} P=O^{\prime} P^{\prime}$, it is related to the $R=O C$ through $R^{2}=r^{2}+Y_{\mathrm{A}}^{2}$. Using Equation (7), we obtain the final reconstruction formula of $R$

$$
R^{2}=X_{\mathrm{A}}^{2}+Y_{\mathrm{A}}^{2}+\left[\tan \left(\frac{\Delta \lambda}{2}\right) X_{\mathrm{A}}+\frac{\Delta X}{\sin \Delta \lambda}\right]^{2} .
$$

\subsection{Displacement Estimation and 3D Reconstruction}

To reduce the computation time, we estimate the value of $\Delta X_{\mathrm{s}}$ around the expected displacement at the photospheric level using the formula (3) in which we use $R=R_{\mathrm{S}}$ where $R_{\mathrm{S}}$ is the photospheric radius. We then use $\Delta X_{\mathrm{S}}$ as a first guess and let the Velociraptor algorithm (Gissot and Hochedez, 2007) find the precise sub-pixel displacement $\Delta X$ around $\Delta X_{\mathrm{s}}$. 


\subsection{Error Formula}

We only consider the variance on $\Delta X$, and neglect the uncertainties on other variables $X_{\mathrm{A}}, Y_{\mathrm{A}}$, and $\Delta \lambda$ since they have significantly lower contribution to the total error. If $V$ is a centered normal random variable, we have the formulas $\mu_{\mathrm{V}^{2}}=\mu_{\mathrm{V}}^{2}+\sigma_{\mathrm{V}}^{2}$ and $\sigma_{\mathrm{V}^{2}}^{2}=4 \sigma_{\mathrm{V}}^{2} \mu_{\mathrm{V}}^{2}+2 \sigma_{\mathrm{V}}^{4}$. Using these formula, and assuming that $\mu_{\Delta \mathrm{X}}=\Delta X$ is the mean value of $\Delta X$, we compute

$$
\mu_{\mathrm{R}^{2}}=X_{\mathrm{A}}^{2}+Y_{\mathrm{A}}^{2}+\frac{1}{\sin (\Delta \lambda)^{4}}\left[\left(2 \sin (\Delta \lambda / 2)^{2} X_{\mathrm{A}}+\Delta X\right)^{2}+\sigma_{\Delta \mathrm{x}}^{2}\right],
$$

and

$$
\sigma_{\mathrm{R}^{2}}^{2}=\frac{2 \sigma_{\Delta \mathrm{x}}^{2}}{\sin (\Delta \lambda)^{8}}\left[\left(2 \sin (\Delta \lambda / 2)^{2} X_{\mathrm{A}}+\Delta X\right)^{2}+2 \sigma_{\Delta \mathrm{x}}^{2}\right] .
$$

If we suppose that $R$ as calculated from (8) behaves like a random, normallydistributed variable of mean value $\mu_{\mathrm{R}}$ and variance $\sigma_{\mathrm{R}}^{2}$, and that

$$
\mu_{\mathrm{R}}=\sqrt{\mu_{\mathrm{R}^{2}}-\sigma_{\mathrm{R}}^{2}} \simeq \sqrt{\mu_{\mathrm{R}^{2}}},
$$

where we assumed that $\mu_{\mathrm{R}^{2}}>>\sigma_{\mathrm{R}}^{2}$, then the variance $\sigma_{\mathrm{R}}^{2}$ depends on $\sigma_{\mathrm{R}^{2}}^{2}$ through the relation

$$
\sigma_{\mathrm{R}^{2}}^{2}=4 \sigma_{\mathrm{R}}^{2} \mu_{\mathrm{R}}^{2}+2 \sigma_{\mathrm{R}}^{4} .
$$

After solving the second degree equation (9), we obtain the formula

$$
\sigma_{\mathrm{R}}^{2}=\frac{1}{4}\left[\sqrt{16 \mu_{\mathrm{R}}^{4}+8 \sigma_{\mathrm{R}^{2}}^{2}}-4 \mu_{\mathrm{R}}^{2}\right] \simeq \frac{\sigma_{\mathrm{R}^{2}}^{2}}{4 \mu_{\mathrm{R}}^{2}},
$$

assuming that $\sigma_{\mathrm{R}^{2}}^{2}<<\mu_{\mathrm{R}}^{4}$. This formula gives the variance of $R$.

\section{STEREO-EUVI Application: Data Reduction and First Results}

\subsection{Description of the STEREO Dataset}

We choose a set of EUVI images observed in $30.4 \mathrm{~nm}$ passband observed on 19 May 2007 at times 12:41:45 (top), 12:51:45 (middle) and 13:01:45 (bottom), which have a lossy compression level ICER5. We process them to reconstruct the radius $R$ at each pixel in the image EUVI-A. The stereo separation angle is $\Delta \lambda=8.6 \mathrm{deg}$.

\subsection{Pre-registration: Limb Fitting and Rotation Correction}

In order to have correct pointing to the Sun centre, we run the secchi_prep.pro IDL routine on a pair of fits images that allows a very high quality pointing. Now images EUVI-A and EUVI-B have their centre pointing to the Sun centre $O$. We then apply the procedure scc_stereopair.pro so that both images the conditions required in Section 2 (see Figure 1). At the end of this pre-registration step, we apply the formula described in Section 2 and Figure 4. 


\subsection{Displacement Estimation}

From the formula (3) we derive the expected displacement between EUVI-A and EUVI-B images. We then apply the Velociraptor algorithm, which gives the displacement at a sub-pixel precision. A high precision is required on the estimation of the upper chromosphere observed in the $30.4 \mathrm{~nm}$ passband because the maximum expected height of the maximum network altitude is $\approx 10 \mathrm{Mm}$ above the photosphere altitude $(\approx 1.45 \%$ of the solar photospheric radius $)$, while prominences are normally observed in the $10 \mathrm{Mm}-100 \mathrm{Mm}$ altitude range.

\subsection{Depth Estimation}

In this particular sequence of 19 May 2007, a filament located in the NOAA active region AR0956 is erupting from frame 12:51:45. The existence of this filament is confirmed by the $\mathrm{H} \alpha$ observation of 19 May 2007 at 06:36:32 provided by the Kanzelhöhe observatory (see Figure 7). The GOES flare curve indicates that a Cflare is simultaneously observed in the same active region. At the three different times, we reconstruct the full solar disk; Figure 5 shows the maps of relative radius $R$. On the left part are the original images, while the radius maps stand in the right column. The dark values, indicating heights below the surface, are due to unstable displacement estimations. In this sequence of three reconstructions, the erupting filament, indicated by a square region of interest (ROI) in the upper right image of Figure 5. The zooms on this ROI are shown in Figure 6. We plot the heights of points located on the filament in Figure 8 and on line segments across the filament in Figure 9. The 1-sigma error bar provided by the formula (10), using a value of $\sigma_{\Delta \mathrm{X}}=0.5$, are overplotted on the height curves. Figures 8 and 9 show the process during which the filament disappears at the same time that a C-flare is observed in the $30.4 \mathrm{~nm}$ image (Figure 6, middle and bottom figures); it is confirmed by the GOES flux (Figure 7 (b)). During this sequence of images, the filament is rising and expanding as shown in Figure 9. In the middle image (12:51:45), the flare starts after the onset of the filament eruption. In the lower right figure (13:01:45) of Figure 6, the filament has partially broken away. The brighter part of the ribbons of the flare is situated on both sides of the polarity inversion line (PIL) but only in the part of the PIL where no elevated filament is observed, while a less bright ribbon is observed very closely aligned with the erupting filament, possibly because of the projection effect due to the filament elevation. From our analysis, a possible scenario is that the PIL passes between the two ribbon sites where there is no filament, and that in this part of the PIL, a flare occurs and causes the two ribbons as well as post-flare loops observed in images of hotter coronal lines such as $19.5 \mathrm{~nm}$. Along the part of the PIL where the filament is reconstructed, the eruption occurs without ribbons and post-flare loops because there is no plasma heating in the reconnection site of the erupting filament. In a future work, the filament height estimations will be compared with critical heights of eruption defined and analysed in Filippov and Den, Filippov and Koutchmy (2001, 2002). The time cadence (10 minutes) limits the measurement of the velocity of the erupting filament. Despite this limitation, the present study enables the $3 \mathrm{D}$ reconstruction of the on-disc eruption. 

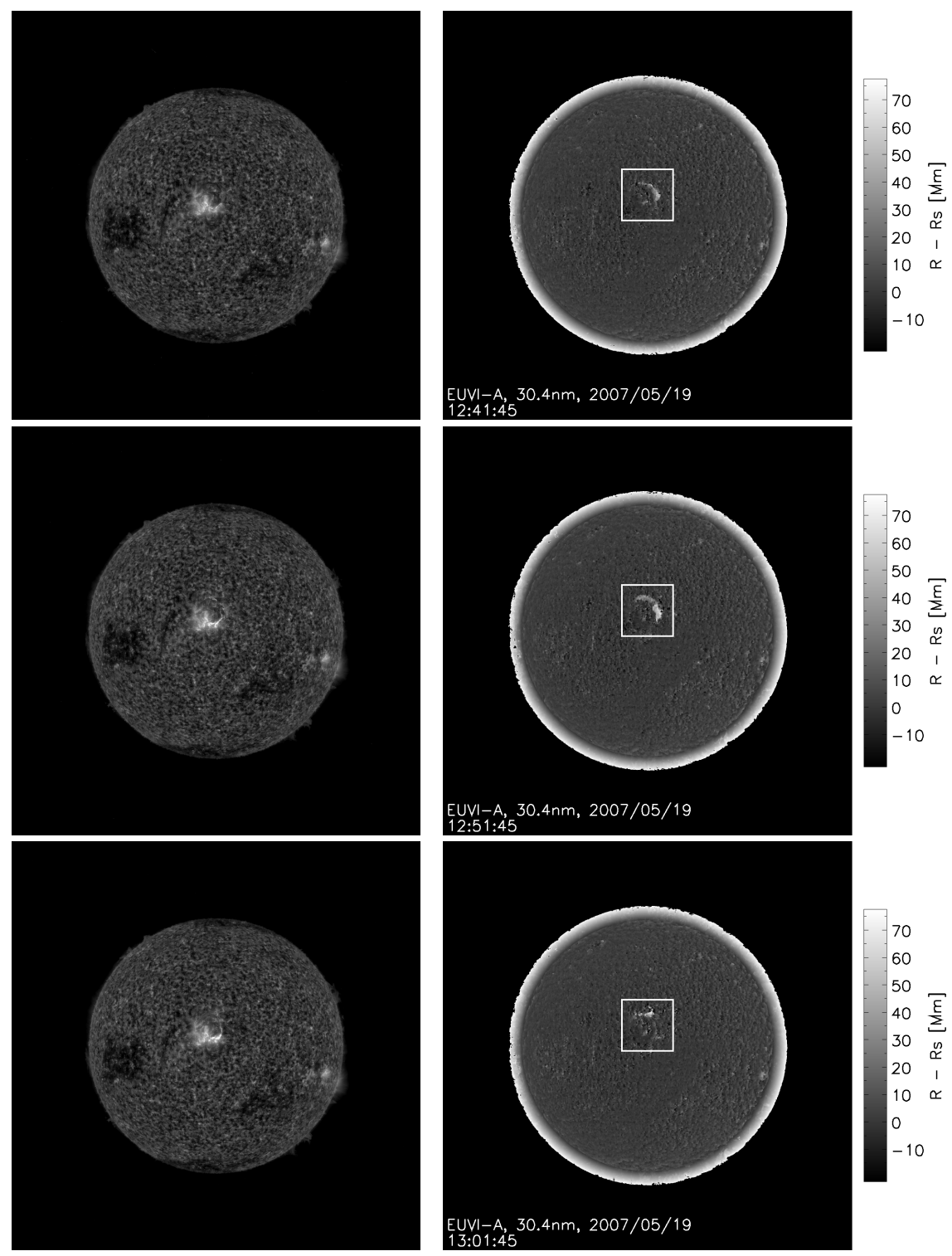

Figure 5. Depth map of the EUVI-A stereoscopic reconstruction. Left: original EUVI-A images observed at times on 19 May 2007 at times 12:41:45 (top), 12:51:45 (middle) and 13:01:45 (bottom). Right: corresponding maps of radius estimations, showing the height $R-R_{\mathrm{S}}$ above limb. 

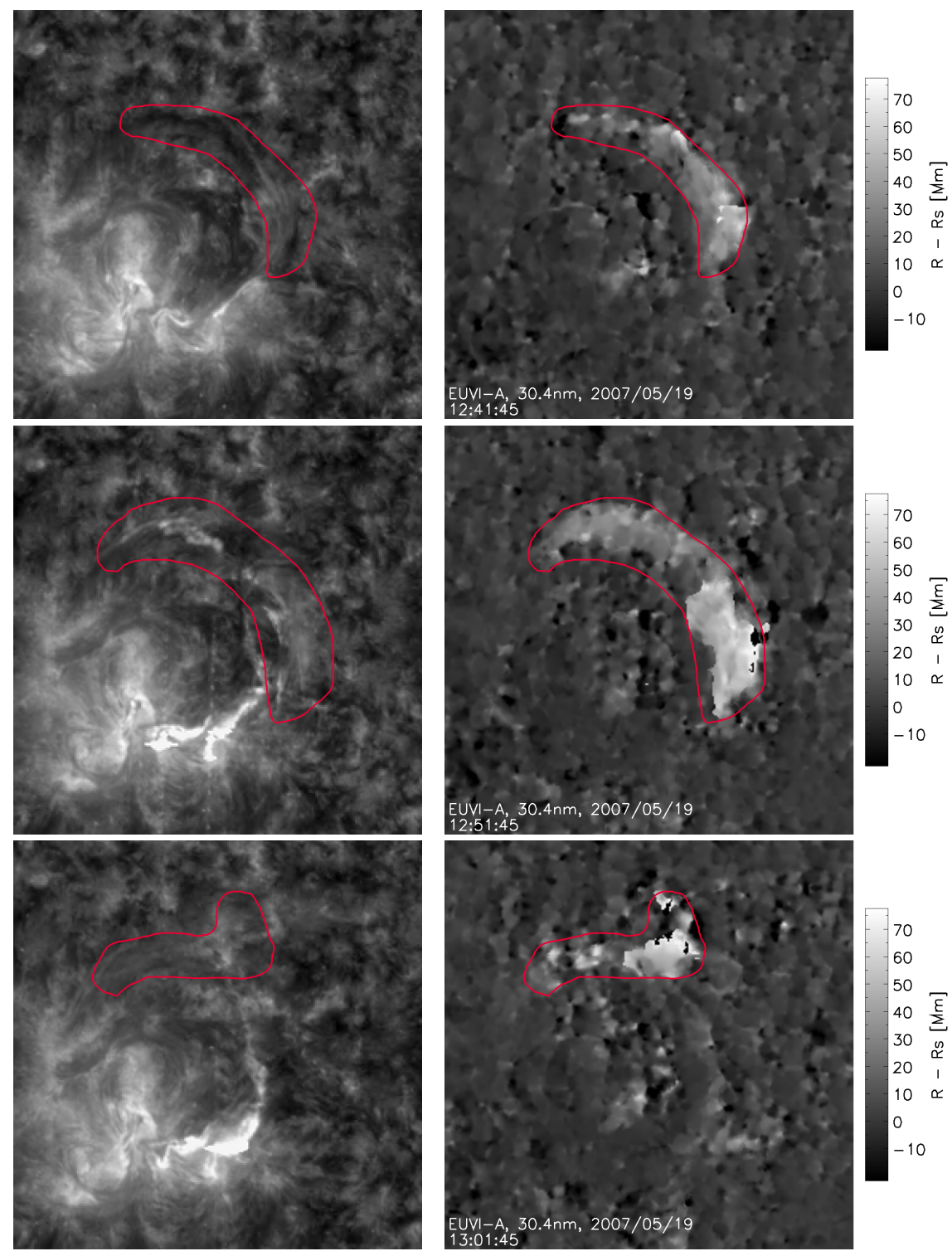

Figure 6. Depth map of the EUVI-A stereoscopic reconstruction. Left: zoom on original EUVI-A images observed at times on 19 May 2007 at times 12:41:45, 12:51:45 and 13:01:45. Right: zoom on corresponding maps of radius estimations, showing the height $R-R_{\mathrm{s}}$ above the limb. 


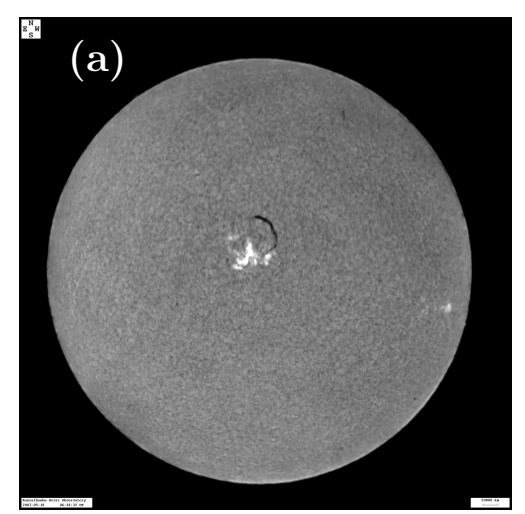

(b)

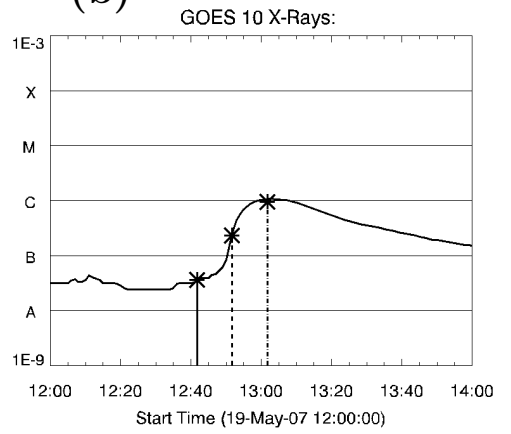

Figure 7. The presence of the filament is also observed in the Kanzelhöhe H $\alpha$ image on 19 May 2007 at 06:36:32 UT in panel (a). The presence of the C-flare is confirmed by the GOES flux in panel (b).
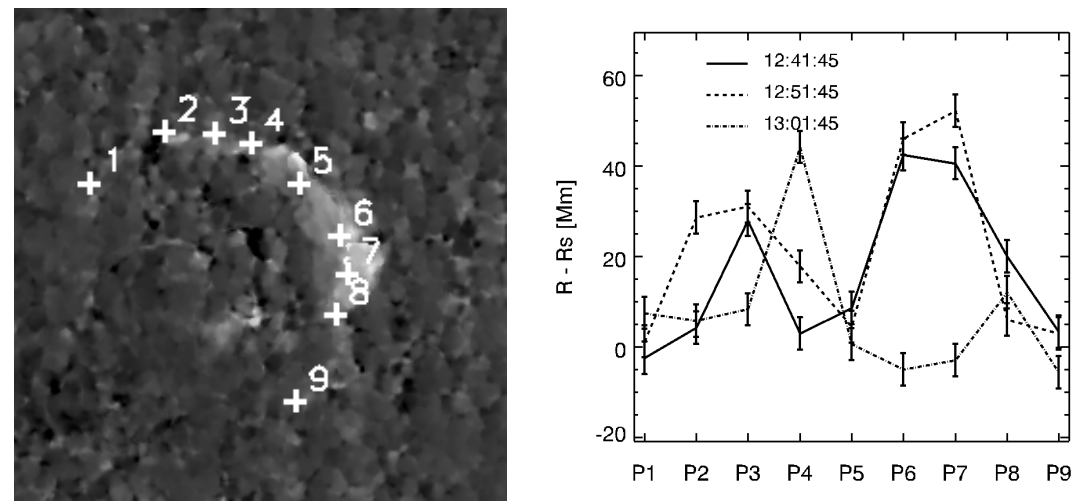

Figure 8. Height above the limb $\left(R-R_{\mathrm{S}}\right)$ for pixels 1 to 9 .

\section{Conclusion}

We have presented our method of stereoscopic reconstruction using pairs of EUVI images provided by the two STEREO spacecrafts. We have first established in the heliocentric coordinate system the formula that provides, given the separation angle and a preprocessing step, the expected displacement between two pixels at the photospheric radius. After inversion of this formula, we derive the stereoscopic reconstruction formula that gives the radius of solar features given the estimated apparent displacement between the two STEREO frames. We use the former formula to calculate the expected displacement at the photospheric level in order to lower the computation time. In the sequence of 19 May 2007, the study of the altitude of the eruptive filament is achieved simultaneously with the observation of a C-flare. 


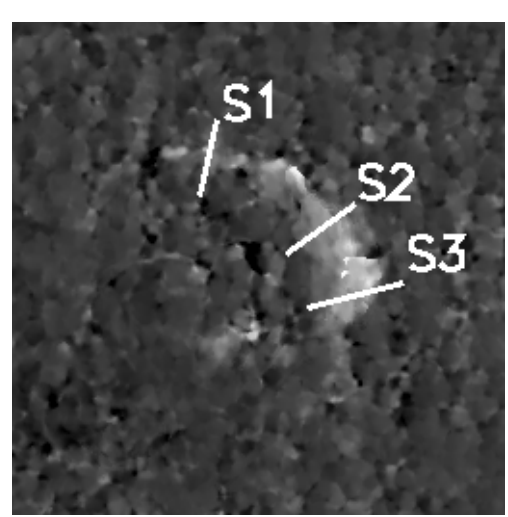

(S1)

$(\mathrm{S} 2)$

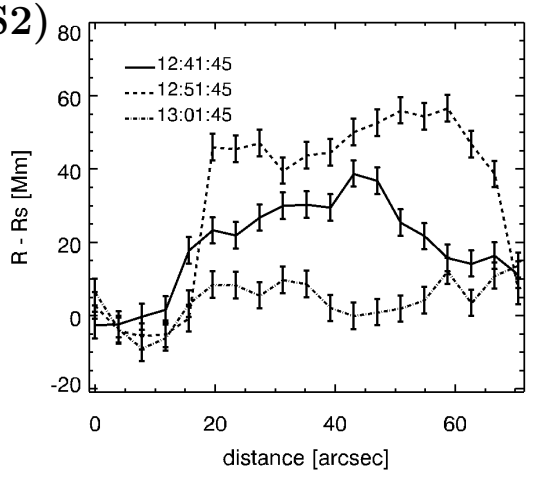

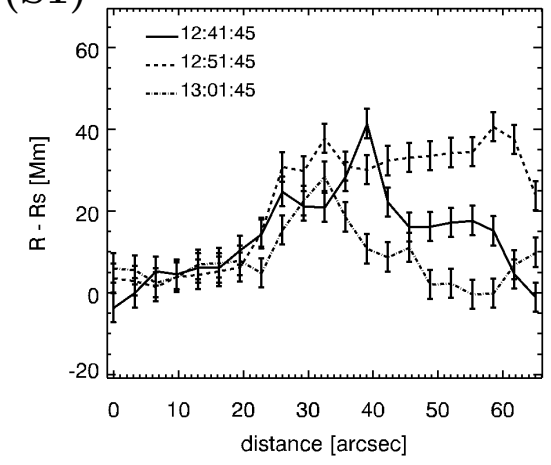

(S3)

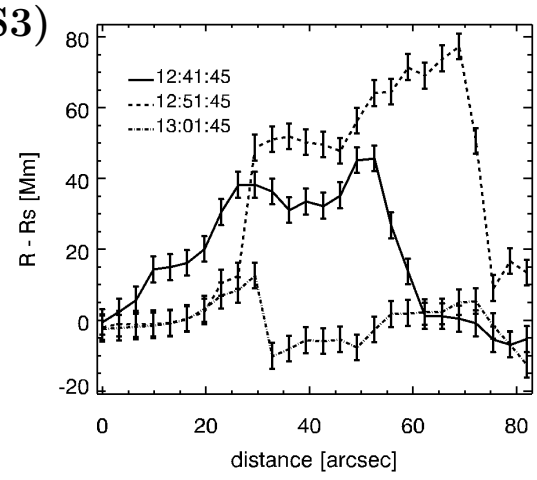

Figure 9. Height variations of segments $\mathrm{S} 1, \mathrm{~S} 2$ and $\mathrm{S} 3$ above the limb $\left(R-R_{\mathrm{S}}\right)$.

In a forthcoming paper, we will process calibration images showing solar-like textures generated on a sphere using the work of Chainais and Li, Chainais $(2005,2007)$, to calibrate the error prediction method developed in Gissot and Hochedez (2007), and improve the value $\sigma_{\Delta \mathrm{x}}=0.5$ in order to assess with precision the error on our radius measurement; we expect to be able to measure precisely the local radius of the Sun in the $30.4 \mathrm{~nm}$ upper chromosphere, in order for instance to assess the altitude of the chromospheric network. This will also allow us to study the altitude of quiescent and eruptive filaments and their evolution along the year 2007 of STEREO data, and study the mass and 3D motion estimation of the erupting filament.

Acknowledgements The authors acknowledge the support from the Belgian government through ESA-PRODEX. STEREO is a NASA mission. This work has also been supported by a France-Belgium Hubert Curien grant "Tournesol (Communauté francophone)". The $\mathrm{H} \alpha$ image is provided by the Kanzelhöhe solar observatory (KSO). We also thanks Andrei Zhukov, Marilena Mierla, and Luciano Rodriguez for fruitful discussions, as well as the anonymous reviewer for his precious comments. 


\section{References}

Brajša, R., Wöhl, H., Vršnak, B., Ruždjak, V., Clette, F., Hochedez, J.-F., and Roša, D.: 2004, Astron. Astrophys., 414, 707

Chainais, P., Li, J.-J.: 2005, In Actes du 20eme colloque GRETSI, Proc. of GRETSI'2005, Presses universitaires de Louvain, 541.

Chainais, P.: 2007, IEEE Transactions On Pattern Analysis and Machine Intelligence, 29.

Delaboudiniére, J.P., Artzner, G.E., Brunaud, J., Gabriel, A.H., Hochedez, J.F., Millier, F., et al.: 1995, Solar Phys. 162, 291.

Feng, L., Inhester, B., Solanki, S. K., Wiegelmann, T., Podlipnik, B., Howard, R. A., and Wuelser, J.-P.: 2007, Astrophys. J. L., 671, L205.

Filippov, B. P., and Den, O. G.: 2001, J. Geophys. Res., 106, 25177.

Filippov, B., and Koutchmy, S.: 2002, Solar Phys., 208, 283.

Gissot, S. F., and Hochedez, J.-F.: 2007, Astron. Astrophys., 464, 1107.

Gissot, S., Hochedez J.-F., Dibos F., Brajša R., Jacques L., Berghmans D., Zhukov A., Clette F., Wöhl H., and Antoine J.-P.: 2003, In Wilson, A. (ed.), Solar Variability as an Input to the Earth's Environment, ESA SP-535, 853.

Inhester, B.: 2006, ArXiv Astrophysics e-prints, arXiv:astro-ph/0612649.

Inhester, B., Feng, L., and Wiegelmann, T.: 2007, Solar Phys., 134.

Kaiser, M. L., Kucera, T. A., Davila, J. M., St. Cyr, O. C., Guhathakurta, M., and Christian, E.: 2007, Space Science Reviews, 136, 5.

Lucas, B.D., and Kanade, T.: 1981, Proc. of the 7th International Joint Conference on Artificial Intelligence (IJCAI'81), 674.

Rosa, D., Vrsnak, B., Bozic, H., Brajsa, R., Ruzdjak, V., Schroll, A., Wöhl, H.: 1998, Solar Phys. 237, 179.

Vršnak, B., Rošd, A., Božić, H., Brajša, R., Ruždjak, V., Schroll, A., Wöhl, H.: 1999, Solar Phys., 185, 207 
SPGissot2008v1.3.tex; 16/07/2008; 18:57; p.14 